\title{
New density profile and structural parameters of the complex stellar system Terzan $5^{1}$
}

\author{
B. Lanzoni' ${ }^{2}$ F.R. Ferraro ${ }^{2}$, E. Dalessandro ${ }^{2}$, A. Mucciarelli², G. Beccari ${ }^{3}$, P. Miocchi², M. \\ Bellazzini $^{4}$, R.M. Rich ${ }^{5}$, L. Origlia ${ }^{4}$, E. Valenti ${ }^{6,7}$, R.T. Rood ${ }^{8}$, S.M. Ransom ${ }^{9}$ \\ ${ }^{2}$ Dipartimento di Astronomia, Università degli Studi di Bologna, via Ranzani 1, I-40127 \\ Bologna, Italy \\ ${ }^{3}$ ESA, Space Science Department, Keplerlaan 1, 2200 AG Noordwijk, Netherlands \\ 4 INAF- Osservatorio Astronomico di Bologna, Via Ranzani, 1, 40127 Bologna, Italy \\ 5 Department of Physics and Astronomy, Math-Sciences 8979, UCLA, Los Angeles, CA \\ 90095-1562, USA \\ ${ }^{6}$ P. Universidad Catolica de Chile, Departamento de Astronomia y Astrofisica, Casilla 306, \\ Santiago 22, Chile \\ 7 European Southern Observatory, Alonso de Cordova 310\%, Vitacura, Santiago, Chile \\ ${ }^{8}$ Astronomy Department, University of Virginia, P.O. Box 400325, Charlottesville, VA, \\ 22904, USA \\ 9 National Radio Astronomy Observatory, Charlottesville, VA 22903, USA
}

12 Mai, 2010

\begin{abstract}
Terzan 5 is a globular cluster-like stellar system in the Galactic Bulge which has been recently found to harbor two stellar populations with different iron content and probably different ages (Ferraro et al. 2009). This discovery suggests that Terzan 5 may be the relic of a primordial building block which contributed to the formation of the Galactic Bulge. Here we present a re-determination of the structural parameters (center of gravity, density and surface brightness profiles, total luminosity and mass) of Terzan 5, as obtained from the combination of high-resolution ( $E S O-\mathrm{MAD}$ and $H S T$ ACS-WFC) and wide-field (ESO-WFI) observations. We find that Terzan 5 is significantly less concentrated and more massive than previously thought. Still it has the largest collision rate of any stellar aggregate in the Galaxy. We discuss the impact of these findings on the exceptional population of millisecond pulsars harbored in this stellar system.
\end{abstract}


Subject headings: Globular clusters: individual (Terzan 5); stars: evolution

\section{INTRODUCTION}

Terzan 5 is commonly catalogued as a globular cluster (GC) located in the inner Bulge of our Galaxy. It is difficult to observe because it is heavily reddened, with an average color excess $E(B-V)=2.38$ (Barbuy et al. 1998; Valenti et al. 2007). Not only is the reddening large, but it strongly depends on the line of sight (differential reddening; see Ortolani et al. 1996, Valenti et al. 2007). Terzan 5 has an exceptionally large population of millisecond pulsars (MSPs). Indeed, the 33 MSPs detected so-far in Terzan 5 amount to about $25 \%$ of the entire sample of known MSPs in Galactic GCs (Ransom et al. 2005; see the updated list at http: //www.naic.edu/pfreire/GCpsr.html)

As part of a project (Ferraro et al. 2001, 2003; Cocozza et al. 2008) aimed at studying the properties of stellar populations harboring MSPs, we obtained a set of high-resolution images of Terzan 5 in the $K$ and $J$ bands using the multi-conjugate adaptive optics (AO) demonstrator MAD (Marchetti et al. 2007) temporally installed at the European Southern Observatory (ESO) Very Large Telescope (VLT). The $(K, J-K)$ color-magnitude diagram (CMD) obtained from these observations led to the discovery of two well-defined red horizontal branch $(\mathrm{HB})$ clumps, clearly separated in luminosity $(\delta K \sim 0.3)$ and color (see Ferraro et al. 2009, hereafter F09; also see Figure 1). A prompt spectroscopic follow-up demonstrated that the two populations have the same radial velocity (hence they belong to the same stellar systems) and their metal content is different: $[\mathrm{Fe} / \mathrm{H}] \simeq-0.2$ and $[\mathrm{Fe} / \mathrm{H}] \simeq+0.3$ for the fainter and the brighter group, respectively. These findings and the comparison with theoretical stellar isochrones confirm the existence of two distinct stellar populations in Terzan 5 and suggest that they possibly have been generated by two bursts of star formation with a time separation of a few $(\approx 6)$ Gyrs. While the age gap can be reduced by invoking a difference in the helium content of the two populations (D'Antona et al. 2010), the iron enrichment and the spatial segregation of the brightest clump, together with the extraordinary amount of MSPs found in Terzan 5, indicate that this system probably experienced a particularly troubled formation and evolutionary history (see Sect. 5). Terzan 5 is the first a GC-like system in the Galactic Bulge found to have a spread in the iron content and it could be the

\footnotetext{
${ }^{1}$ Based on observations collected at the European Southern Observatory, Cerro Paranal (during the Science Demonstration of MAD) and La Silla (under proposal 278.D-5067), Chile. Also based on observations with the NASA/ESA HST, obtained at the Space Telescope Science Institute, which is operated by AURA, Inc., under NASA contract NAS5-26555.
} 
relic of one of the building blocks that contributed to the formation of the Bulge. Indeed the discovery might represent the observational evidence that even the innermost part of galactic spheroids form (at least partially) by the accretion/merging of small, previously formed and internally evolved stellar systems (e.g., Immeli et al. 2004).

In this paper we present the accurate re-determination of Terzan 5 structural parameters (surface density and surface brightness profiles, total luminosity, collision rate, etc.), obtained from a combination of high-resolution and wide-field observational data. These parameters provide basic information for a deeper understanding of the origin and the evolution of this puzzling system.

\section{THE DATA}

The photometric dataset used in the present work consists of high-resolution and widefield images obtained, respectively, with MAD at the ESO-VLT and the Advanced Camera for Survey (ACS) on board the Hubble Space Telescope (HST), and with the Wide Field Imager (WFI) at the 2.2m ESO-MPI telescope.

1. The MAD-dataset consists of a set of short (2-minute long), AO-corrected exposures secured through the $K$ and $J$ filters in August 2008, as part of a MAD science demonstration project (P.I. Ferraro). From all these images, we chose and analysed the highest quality ones: the full width at half maximum (FWHM) measured for the selected $K$ and $J$ images is $0.1^{\prime \prime}$ and $0.24^{\prime \prime}$, respectively, just slighly larger than the diffraction limit. More importantly the FWHM is extremely stable over the entire $\left(1^{\prime} \times 1^{\prime}\right)$ MAD field of view (FOV), fully demonstrating the potentiality of the multi-conjugate $\mathrm{AO}$ correction.

2. The HST-dataset consists of deep ACS Wide Field Camera (WFC) images obtained through filters F606W (a broad $V$ ) and F814W $(I)$, with total exposure times of $340 \mathrm{~s}$ and 360 s, respectively (Prop. 9799, P.I. Rich).

3. The Wide field-dataset consists of multiple $V$ and $I$ images $(4 \times 120 \mathrm{~s}$ exposures each) obtained with the ESO-WFI at La Silla (Chile) and retrieved from the ESO Science Archive (Prop. 278.D-5067(A); P.I. Bassa). The WFI is a mosaic of 8-CCD chips which combines wide-field $\left(\mathrm{FOV}=33^{\prime} \times 34^{\prime}\right)$ and reasonably high-resolution capabilities (pixel size of $\left.\sim 0.24^{\prime \prime}\right)$. The core of the cluster is roughly centered on CCD 7 and the images allow to sample the entire cluster extension (see Sect. 4).

The point spread function (PSF) for each image has been modelled on several bright and isolated stars, by using the DAOPHOTII/PSF routine (Stetson 1987). Then PSF- 
fitting photometry of the MAD dataset has been perfomed independently on the $K$ and $J$ best images, using DAOPHOTII/ALLSTAR. We then used DAOPHOTII/MONTAGE2 procedure to produce a stacked master frame combining the optical images in all the available filters (F606W and F814W for the ACS observations, and $V$ and $I$ for the WFI dataset). This method allowed us to obtain, for each optical dataset, a single high $\mathrm{S} / \mathrm{N}$ image, cleaned from specific detector defects (i.e. hot pixels) and other spurious sources like cosmic rays etc. A master star list has been searched at a $4 \sigma$ detection limit on this reference frame and it has then been used as input for ALLFRAME (Stetson 1994), which simultaneously determines the brightness of the stars in all the frames, while enforcing one set of centroids and one transformation between all the images. Finally, the magnitudes obtained for each star have been normalized to a reference frame and averaged together, and the photometric error was derived from the standard deviation of the repeated measures.

The star positions in the WFI sample were placed on the absolute astrometric system by using more than twenty thousand stars in common with the new astrometric Two Micron All Sky Survey (2MASS) catalogue 2 . Then the $\sim 6000$ stars in common between the WFI and the ACS datasets have been used as secondary astrometric standards for placing the ACS sample on the absolute astrometric system, and the same has been done for the $\sim$ 9000 stars in common between the ACS and the MAD datasets, following the procedure described, e.g., in Lanzoni et al. (2007). The final astrometric accuracy of all the samples is of the order of $\sim 0.2^{\prime \prime}$ both in right ascension $(\alpha)$ and in declination $(\delta)$. The near-infrared instrumental magnitudes have been reported to the 2MASS photometric system by using the stars in common with the catalog of Valenti et al. (2007). The optical magnitudes have been transformed and homogenized by using a sample of stars in common with Ortolani et al. (1996).

The CMDs obtained for the three datasets are shown in Figure1, The two HB clumps discussed by F09 are clearly visible in the MAD dataset and partially distinguishable in the ACS sample, while the field contamination (mainly from the Galactic Bulge and Disk) is progressively more important at increasing distance $(r)$ from the cluster center. The optical CMD clearly shows how Terzan 5 is strongly affected by differential reddening. We are currently constructing a detailed differential reddening map in the direction of this stellar system (Mucciarelli et al. 2010, in preparation), and preliminary results suggest that the reddening variation can be as high as $\delta E(B-V)=0.6$.

\footnotetext{
${ }^{2}$ Available at http://irsa.ipac.caltech.edu.
} 


\section{CENTER OF GRAVITY}

F09 determined the center of gravity $\left(C_{\text {grav }}\right)$ of Terzan 5 by using the absolute positions of individual stars detected in the MAD sample. As a check, here we have recomputed $C_{\text {grav }}$ by exploiting the ACS dataset, with a cut in magnitude $I=21$ needed to avoid spurious effects due to incompleteness in the very inner regions of the cluster. We found that, within the uncertainty $\left(\sim 0.5^{\prime \prime}\right.$ in both $\alpha$ and $\left.\delta\right)$, the two determinations are coincident, and we therefore confirm that gravity center of Terzan 5 is located at (F09): $\alpha_{\mathrm{J} 2000}=17^{\mathrm{h}} 48^{\mathrm{m}} 4.85^{\mathrm{s}}$, $\delta_{J 2000}=-24^{\circ} 46^{\prime} 44.6^{\prime \prime}$. This is $\sim 1^{\prime \prime}$ north-west from the photometric centre quoted by Harris (1996), and $\sim 1^{\prime \prime}$ north-east from that of Cohn et al. (2002).

To further check the reliability of our determination we also used a different approach. This relies on the algorithm defined by Casertano \& Hut (1985) to calculate the "density center" of a stellar system (for more details see Miocchi et al. 2010, in preparation). As a first step, for all the $N$ stars located within a circle of a given radius, centered at an initially guessed position, the local (surface) density $\rho_{i}$ around the position of the $i$ th star is evaluated as the inverse of its squared distance from the sixth nearest star. Then the cluster center is computed as the density-weighted average of the $N$ star positions. The evaluation is repeated by centering the circle at the last found points and the iteration stops when the distance between the new and the previous determinaton is smaller than a given value (usually $0.01^{\prime \prime}$ ). The density center thus computed agrees with the value quoted above well within the uncertainties.

Finally, we also checked whether the two HB populations share the same gravity centre. To this purpose we have selected them from the ACS dataset, on the basis of the star position in the $(I, V-I)$ CMD (see Supplementary Figure 1 in F09). The projected spatial distribution of the two selected samples is plotted in Figure 2 and clearly shows that the bright HB population is more centrally concentrated than the faint one, in agreement with what found by F09 from the MAD dataset. The barycentres of the two HB populations seem to be different, with the faint $\mathrm{HB}$ centre being located $\approx 3^{\prime \prime}$ south-east from that of the bright HB stars, which almost coincides with the cluster gravity centre quoted above. We stress however that, while the optical selection allowed us to improve the statistics and increase the radial coverage of the samples at most, it does not guarantee a proper separation of the two groups of stars. Hence, before confirming such a finding it is necessary to perform a more robust and clean selection of the two HB populations, based on (presently not available) near-infrared data covering a much larger area than the MAD FOV. 


\section{PROJECTED DENSITY AND SURFACE BRIGHTNESS PROFILES}

We have determined the projected density profile of Terzan 5 using direct star counts on the available datasets, which cover the entire radial extent of the cluster. For the innermost part of the profile we have exploited the high-resolution (MAD and ACS) datasets, while for $r>100^{\prime \prime}$ we have used the WFI sample and complementary data from the 2MASS survey, thus covering radial distances out to $r=1700^{\prime \prime}$. To avoid incompleteness biases, different limiting magnitudes have been adopted for the four datasets: $K=13, I=20, I=19$, and $K=12.5$ for the MAD, ACS, WFI, and 2MASS samples, respectively. By also adopting the colour cuts $(V-I)>3.4$ and $(J-K)>1.3$ we have excluded from the analysis the contribution of the stars belonging to the Galactic disk main sequence. With these limits we have computed the four portions of the density profile corresponding to each dataset 3 In total more than 50,000 stars were used to construct the star density profile. Following the procedure described in Ferraro et al. (1999) we have divided the samples in concentric annuli centered on $C_{\text {grav }}$, and each annulus has been split into an adequate number of subsectors. The number of stars lying within each sub-sector was counted, and the star surface density was obtained by dividing these values by the corresponding sub-sector areas. The stellar density in each annulus was then obtained as the average of the sub-sector densities, and the standard deviation was adopted as the uncertainty. Then, the radial annuli in common between the different samples have been used to shift and join the various portions of the profile. The overall projected density profile thus obtained is shown in Figure 3 (empty squares), with the abscissae corresponding to the mid-point of each radial bin. The outermost $\left(r \gtrsim 175^{\prime \prime}\right)$ measures from the WFI and 2MASS samples have an almost constant value, and their average has been used to estimate the Galactic Bulge and Disk contamination level. The subtraction of this background yields the profile shown in the figure as filled dots. The derived density profile is well fit all over its radial extension by an isotropic, single-mass King model (King 1966) with core radius $r_{c}=9.0^{\prime \prime}$, half-mass radius $r_{h}=31^{\prime \prime}$, tidal radius radius $r_{t}=277^{\prime \prime}=4.6^{\prime}$, and intermediate concentration $(c=1.49)$. While the size of the core radius is consistent with the most recent determination $\left(r_{c}=7.9^{\prime \prime}\right.$; Cohn et al. 2002), the concentration is significantly smaller than that $(c \approx 2)$ suggested by those authors, and the ratio between the core and the half-mass radius is a factor of two larger in our case.

Exploiting the exceptional quality of the available datasets, we have also computed the

\footnotetext{
${ }^{3}$ While different limits have been (necessarily) adopted for the four datasets, the mass of the sampled stars is roughly constant (comparable to the that of the main sequence turn off stars). Hence any mass segregation effect on the four different portions of the profile is expected to be negligible. This is further confirmed by the agreeement found between the number density and the surface brightness profiles (see below).
} 
surface brightness (SB) profiles by aperture photometry on the MAD and ACS images. The $\mathrm{SB}$ values were computed as the sum of the photon counts in each pixel, divided by the sampled area in any given radial annulus. The counts have been converted to a magnitude scale and then calibrated using a relation derived by performing aperture photometry on a number of high S/N isolated stars. The resulting SB profile, obtained for the inner $\sim 100^{\prime \prime}$ from the center, after proper subtraction of the background level and of a few artefacts due to saturated stars, is shown in three different filters in Figure 4. These profiles are well fit by the same King model derived from the projected density distribution, thus confirming that the samples of resolved stars used above are not affected by radial variations of the completeness and are properly selected. The values of the central SB measured in the three photometric bands are listed in Table 1, together with all the relevant parameters derived for Terzan 5 .

\section{DISCUSSION}

The star density and SB profiles can be used to derive the integrated luminosity of the cluster. From the best-fit King model we estimate that the percentage of cluster light within regions of radius $r=15^{\prime \prime}, 18^{\prime \prime}$, and $20^{\prime \prime}$ are roughly $30 \%, 36 \%$ and $40 \%$, respectively. Using aperture photometry on the MAD images, we obtain integrated-light values of $K(r<$ $\left.15^{\prime \prime}\right)=3.44, K\left(r<18^{\prime \prime}\right)=3.3$ and $K\left(r<20^{\prime \prime}\right)=3.2 \mathrm{mag}$, respectively. Adopting the color excess $E(B-V)=2.38$, the distance modulus $(m-M)_{0}=13.87$ (Valenti et al. 2007, corresponding to a distance of $d=5.9 \pm 0.5 \mathrm{kpc}$ ) and the bolometric correction $B C_{K}=2.4$ appropriate for a population of intrinsic color $(J-K)_{0}=0.8$ (see Montegriffo et al. 1998), we estimate that the corresponding bolometric luminosity in the considered regions is: $L_{\mathrm{bol}}\left(r<15^{\prime \prime}\right)=3 \times 10^{5} L_{\odot}, L_{\mathrm{bol}}\left(r<18^{\prime \prime}\right)=3.4 \times 10^{5} L_{\odot}$ and $L_{\mathrm{bol}}\left(r<20^{\prime \prime}\right)=3.7 \times 10^{5} L_{\odot}$. Considering the fraction of light sampled in each region we find that the total luminosity of the system is $L_{\mathrm{bol}}=9.5 \pm 0.3 \times 10^{5} L_{\odot}$.

An independent estimate of the total luminosity of the stellar system can be derived from its stellar population, by using a simple relation (Renzini \& Buzzoni 1986) linking the number of stars $\left(N_{j}\right)$ observed in a given post-main sequence evolutionary stage $j$ and the luminosity of the entire parent cluster $\left(L_{T}\right)$ :

$$
N_{j}=B \times t_{j} \times L_{T}
$$

where $B$ is the specific evolutionary flux (for intermediate/old stellar populations $B=2 \times$ $10^{-11}$ stars $r^{-1} L_{\odot}^{-1}$ ) and $t_{j}$ is the duration of the evolutionary stage. The number of $\mathrm{HB}$ stars counted in the two clumps by F09 is quite large: a total of about 1300 (with 800 
and 500 belonging to the faint and the bright HB clumps, respectively). This population is comparable to (or even larger than) that observed in the largest Galactic GCs, like 47 Tucanae (Beccari et al. 2006a) and NGC 6388 (Dalessandro et al. 2008), and suggests that the overall size of Terzan 5 (in terms of luminosity and mass) is comparable to that of these systems. For a quantitative estimate, we insert the observed number of HB stars in the above relation and adopt $t_{\mathrm{HB}}=10^{8} \mathrm{yr}$. This provides a luminosity of $4 \times 10^{5} L_{\odot}$ and $2.5 \times 10^{5} L_{\odot}$ for the two parent populations, and a total luminosity of $6.5 \times 10^{5} L_{\odot}$ for the entire stellar system. This estimate, which is distance and reddening independent, is quite consistent with the previous one, thus confirming that Terzan 5 has a considerable total luminosity (hereafter we adopt the average value $L_{\text {bol }}=8 \times 10^{5} L_{\odot}$ ), significantly higher than previously thought. By comparison, adopting the values of distance and reddening quoted above and a bolometric correction $L_{\text {bol }} \simeq 1.4 L_{V}$, the total bolometric luminosity corresponding to the integrated magnitude $\left(V_{t}=13.85\right)$ quoted by Harris (1996) would be only $L_{\text {bol }} \simeq 10^{5} L_{\odot}$. The discrepancy is most probably due to the strong (differential) reddening affecting the system, especially in the optical bands. This effect is greatly reduced for our new estimate, since it is based on the observed $K$-band integrated magnitude and the number of HB stars. By assuming a mass-to-light ratio $M / L_{\text {bol }}=3$ (e.g., Maraston 1998), the total stellar mass of this system is $M_{\mathrm{T}} \simeq 2 \times 10^{6} M_{\odot}$.

Verbunt \& Hut (1987) first suggested that the collision rate of Terzan 5 is the highest among the Galactic GCs. We can now re-compute this quantity by adopting the newly determined parameters. Following Verbunt \& Hut (1987), the collisional parameter $(\Gamma)$ for a King-model or virialized system can be computed as: $\Gamma \propto \rho_{0} \times r_{c}^{0.5}$, where $\rho_{0}$ is the central mass density. By using the values obtained above and equation (7) of Djorgovski (1993), we find that the collision parameter of Terzan 5 is between 5 and 10 times higher than that of Liller 1 and of other massive clusters for which structural parameters have been recently redetermined (NGC6388, NGC6266, 47Tuc; Dalessandro et al. 2008; Beccari et al. 2006b; Mapelli et al. 2006, respectively). Hence we confirm that, even with the new structural parameters (suggesting a lower concentration and a larger mass than previously thought), Terzan 5 still has the largest known collision rate of any stellar aggregate in the Galaxy.

The co-existence of two stellar populations with different iron content (and probably ages) suggests that the original mass of Terzan 5 was significantly larger in the past than observed today, large enough to retain the iron-enriched gas that, otherwise, would have been ejected out from the system by the violent supernova (SN) explosions. Indeed, the smallest systems with solid evidences of a spread in the iron content (and ages) are significantly more massive than GCs: the dwarf spheroidal satellites of the Milky Way typically have masses of $\sim 10^{7} M_{\odot}$ (Strigari et al. 2008; see also Battaglia et al. 2008) and, following recent chemodynamical models well reproducing the observations, their initial masses amounted to a few 
$10^{8} M_{\odot}$ (Revaz et al. 2009). While a lower limit of $\sim 10^{7} M_{\odot}$ for the proto-Terzan 5 could also be hazarded following Baumgardt et al. (2008), more detailed and extensive simulations are needed to firmly determine the smallest total mass necessary to retain the SN ejecta.

The exceptionally high metallicity regime of the two stellar populations found in Terzan 5 also suggests a quite efficient enrichment process, that could have a relevant role in the origin of its population of MSPs. In particular, both the iron and the $[\alpha / \mathrm{Fe}]$ abundance ratios measured in Terzan 5 (Origlia \& Rich 2004, Rich et al. 2010, in preparation) show a remarkable similarity with those of the Bulge stars. This strongly suggests that these two structures shared the same star formation and chemical enrichment processes. The many observations of Bulge stars (e.g., Meléndez et al. 2008; Origlia et al. 2008; Ryde et al. 2009, and references therein) indicate that they are all characterized by an old age, a high (close to solar) average metallicity $[\mathrm{Fe} / \mathrm{H}]$, and an $[\alpha / \mathrm{Fe}]$ ratio which is enhanced (due to SNII enrichment) up to a metallicity $[\mathrm{Fe} / \mathrm{H}] \simeq 0$. These constraints suggest a scenario where the dominant stellar population of the Bulge formed early (thus explaining the old age 4 ), rapidly and with high efficiency (from a gas mainly enriched by SNII, thus explaining the $[\alpha / \mathrm{Fe}]$ enhancement up to high iron content:5). Also chemical evolution models (e.g., Ballero et al. 2007; McWilliam et al. 2008) indicate that the abundance patterns observed in the Bulge require a quite high star formation efficiency and an initial mass function flatter than that in the solar neighbourhood, thus to rapidly enrich the gas up to about solar metallicity through an exceptionally large amount of SNII explosions. The assumption of a similar scenario for Terzan 5 would naturally explain its extraordinary population of MSPs, since the expected high number of SNII would produce a large population of neutron stars, most of which would have been retained by the deep potential well of the massive proto-Terzan 5 system. Then the high collision rate could have favoured the formation of binary systems containing neutron stars and promoted the re-cycling process that finally generated the large population of MSPs now observed in Terzan 5. If such a scenario is correct, many more MSPs still wait to be discovered in this system (see also Ransom et al. 2005), the 33 known objects probably being just the tip of the iceberg. Future deeper pulsar searches of Terzan 5, perhaps with larger telescopes such as the Square Kilometer Array, will shed additional light on the nature of this system.

This research was supported by the Agenzia Spaziale Italiana (under contract ASI-

\footnotetext{
${ }^{4}$ Additional episodes of star formation mainly confined in the innermost $(\sim 100 \mathrm{pc})$ region could eventually explain the presence of younger stars (e.g., Blum et al. 2003; Figer et al. 2004).

${ }^{5}$ The $[\alpha / \mathrm{Fe}]-[\mathrm{Fe} / \mathrm{H}]$ relation shows a down-turn at a value of $[\mathrm{Fe} / \mathrm{H}]$ which depends on the star formation rate: the higher the latter, the higher the metallicity at which the down-turn occurs. Such a value is $[\mathrm{Fe} / \mathrm{H}] \simeq-1$ in the Old Halo/Disk, while it is significantly higher $([\mathrm{Fe} / \mathrm{H}] \simeq 0)$ in the Bulge, testifying a much higher star formation rate in this dense environment.
} 
INAF I/016/07/0), by the Istituto Nazionale di Astrofisica (INAF, under contract PRININAF2008) and by the Ministero dell'Istruzione, dell'Università e della Ricerca. RTR is partially supported by STScI grant. RMR is supported by AST-0709479 and GO-9799 from STScI. This research has made use of the ESO/ST-ECF Science Archive facility which is a joint collaboration of the European Southern Observatory and the Space Telescope European Coordinating Facility.

\section{REFERENCES}

Ballero, S. K., Matteucci, F., Origlia, L., \& Rich, R. M. 2007, A\&A, 467, 123

Barbuy, B., Bica, E., \& Ortolani, S. 1998, A\&A, 333, 117

Battaglia, G., Helmi, A., Tolstoy, E., Irwin, M., Hill, V., \& Jablonka, P. 2008, ApJ, 681, L13

Baumgardt, H., Kroupa, P., \& Parmentier, G. 2008, MNRAS, 384, 1231

Beccari, G., Ferraro, F.R., Lanzoni, B., \& Bellazzini 2006a, ApJ, 652, 121

Beccari, G., Ferraro, F. R., Possenti, A., Valenti, E., Origlia, L., \& Rood, R. T., 2006b, AJ, 131,2551

Blum, R. D., Ramírez, S. V., Sellgren, K., \& Olsen, K. 2003, ApJ, 597, 323

Casertano, S., \& Hut, P. 1985, ApJ, 298, 80

Cocozza, G., Ferraro, F. R., Possenti, A., Beccari, G., Lanzoni, B., Ransom, S., Rood, R. T., \& D'Amico, N. 2008, ApJ, 679, L105

Cohn, H. N., Lugger, P. H., Grindlay, J. E., \& Edmonds, P. D. 2002, ApJ, 571, 818

Dalessandro E., Lanzoni, B., Ferraro, F.R., Rood, R.T., Milone, A., Piotto, G., \& Valenti E. et al. 2008, ApJ, 6677, 1069

D’Antona, F., Ventura, P., Caloi, V., D’Ercole, A., Vesperini, E., Carini, R., \& Di Criscienzo, M. 2010, ApJL in press (arXiv:1004.3426)

Djorgovski, S. 1993, ASPC, 50, 373

Ferraro F. R., Messineo M., Fusi Pecci F., De Palo M. A., Straniero O., Chieffi A.,\& Limongi M. 1999, AJ, 118, 1738

Ferraro, F. R., Possenti, A., D’Amico, N., \& Sabbi, E. 2001, ApJ, 561, L93 
Ferraro, F. R., Possenti, A., Sabbi, E., \& D’Amico, N. 2003, ApJ, 596, L211

Ferraro, F. R., et al. 2009, Nature, 462, 483 (F09)

Figer, D. F., Rich, R. M., Kim, S. S., Morris, M., \& Serabyn, E. 2004, ApJ, 601, 319

Harris, W.E. 1996, AJ, 112, 1487

Immeli, A., Samland, M., Gerhard, O., \& Westera, P. 2004, A\&A, 413, 547

King I.R. 1966, AJ, 71, 64

Lanzoni, B., Dalessandro, E., Ferraro, F. R., Mancini, C., Beccari, G., Rood, R. T., Mapelli, M., \& Sigurdsson, S. 2007, ApJ, 663, 1040

Mapelli, M., Sigurdsson, S., Ferraro, F. R., Colpi, M., Possenti, A., \& Lanzoni, B. 2006, MNRAS, 373, 361

Maraston, C. 1998, MNRAS, 300, 872

Marchetti, E., et al. 2007, The Messenger, 129, 8

McWilliam, A., Matteucci, F., Ballero, S., Rich, R. M., Fulbright, J. P., \& Cescutti, G. 2008, AJ, 136,367

Meléndez, J., et al. 2008, A\&A, 484, L21

Montegriffo, P., Ferraro, F. R., Origlia, O., \& Fusi Pecci, F. 1998, MNRAS, 297, 872

Origlia, L., \& Rich, R. M. 2004, AJ, 127, 3422

Origlia, L., Valenti, E., \& Rich, R. M. 2008, MNRAS, 388, 1419

Ortolani, S., Barbuy, B., \& Bica, E. 1996, A\&A, 308, 733

Ransom, S. M., Hessels, J. W. T., Stairs, I. H., Freire, P. C. C., Camilo, F., Kaspi, V. M., \& Kaplan, D. L. 2005, Science, 307, 892

Renzini, A. \& Buzzoni, A., 1986, Spectral evolution of galaxies; Dordrecht, D. Reidel Publishing Co., 195-231

Revaz, Y., et al. 2009, A\&A, 501, 189

Ryde, N., Edvardsson, B., Gustafsson, B., Eriksson, K., Käufl, H. U., Siebenmorgen, R., \& Smette, A. 2009, A\&A, 496, 701 
Stetson, P. B. 1987, PASP, 99, 191

Stetson, P. B. 1994, PASP, 106, 250

Strigari, L. E., Bullock, J. S., Kaplinghat, M., Simon, J. D., Geha, M., Willman, B., \& Walker, M. G. 2008, Nature, 454, 1096

Valenti, E., Ferraro, F. R., \& Origlia, L., 2007, AJ, 133, 1287

Verbunt, F., \& Hut, P. 1987, in Proc. IAU Symp. 125, The Origin and Evolution of Neutron Stars, ed. D. J. Helfand \& J.-H. Huang (Dordrecht: Reidel), 187 

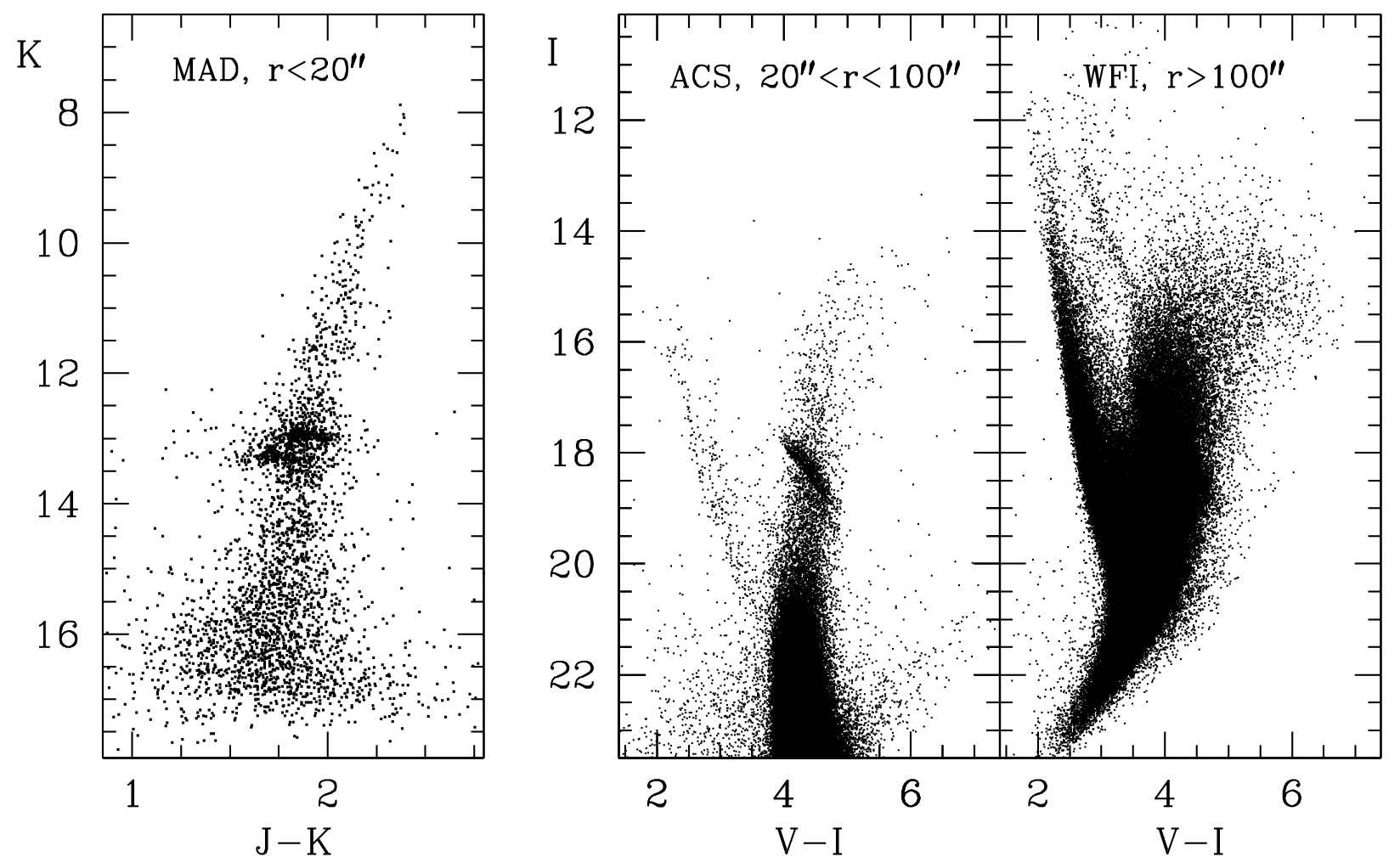

Fig. 1. - Color-magnitude diagrams for the three datasets used in the paper and for different radial regions of the cluster (see labels). The double HB discovered by F09 is clearly visible in the MAD near-infrared CMD, while it is more difficult to distinguish in the ACS optical plane (but see Supplementary Figure 1 in F09). The WFI sample is dominated by field star contamination. 


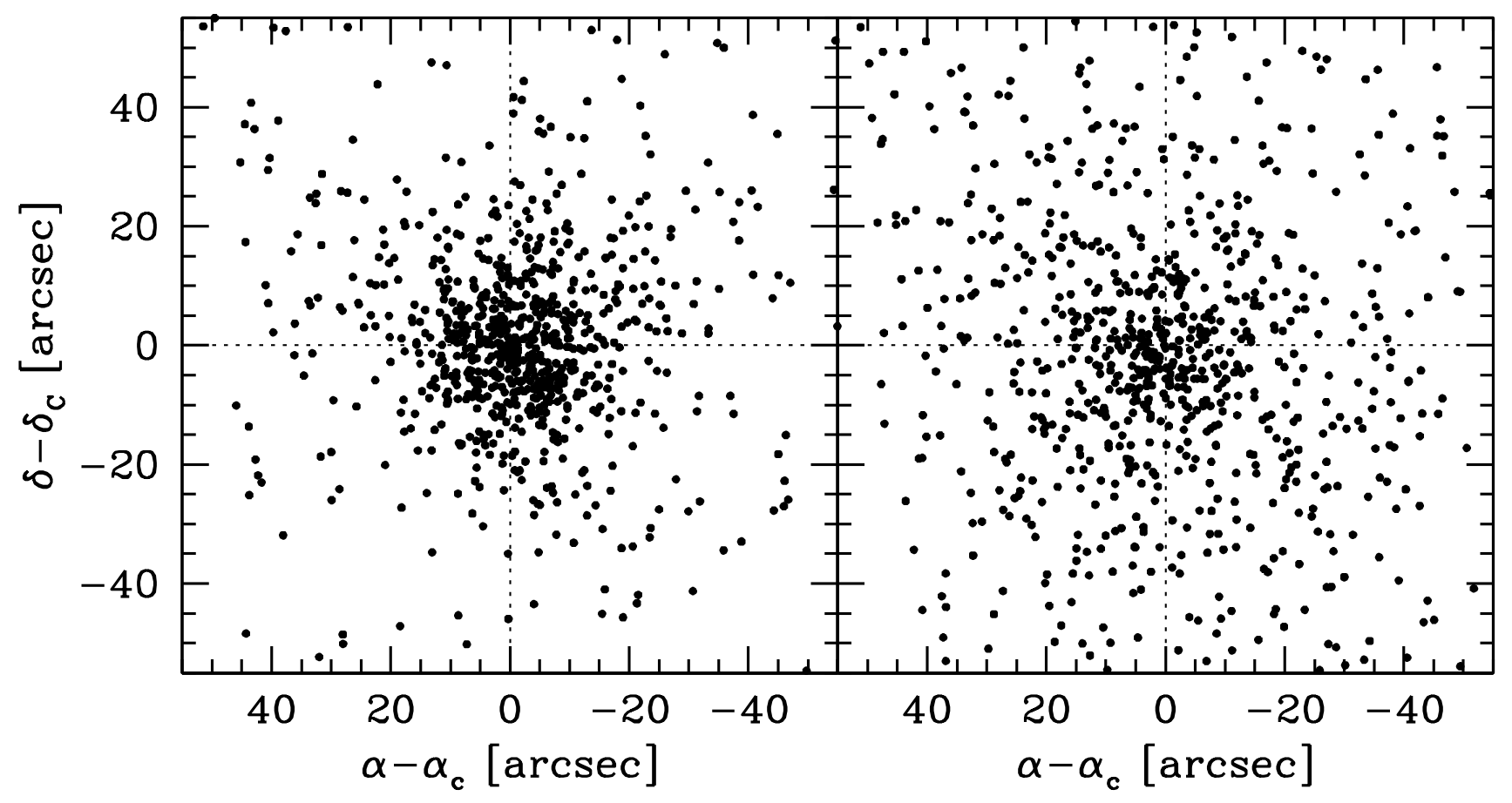

Fig. 2.- Map of the bright (left panel) and faint (right panel) HB populations selected from the ACS dataset on the basis of the star position in the $(I, V-I)$ CMD. The star coordinates $(\alpha$ and $\delta)$ are plotted with respect to those of the cluster gravity centre $\left(\alpha_{\mathrm{c}}\right.$ and $\left.\delta_{\mathrm{c}}\right)$. 


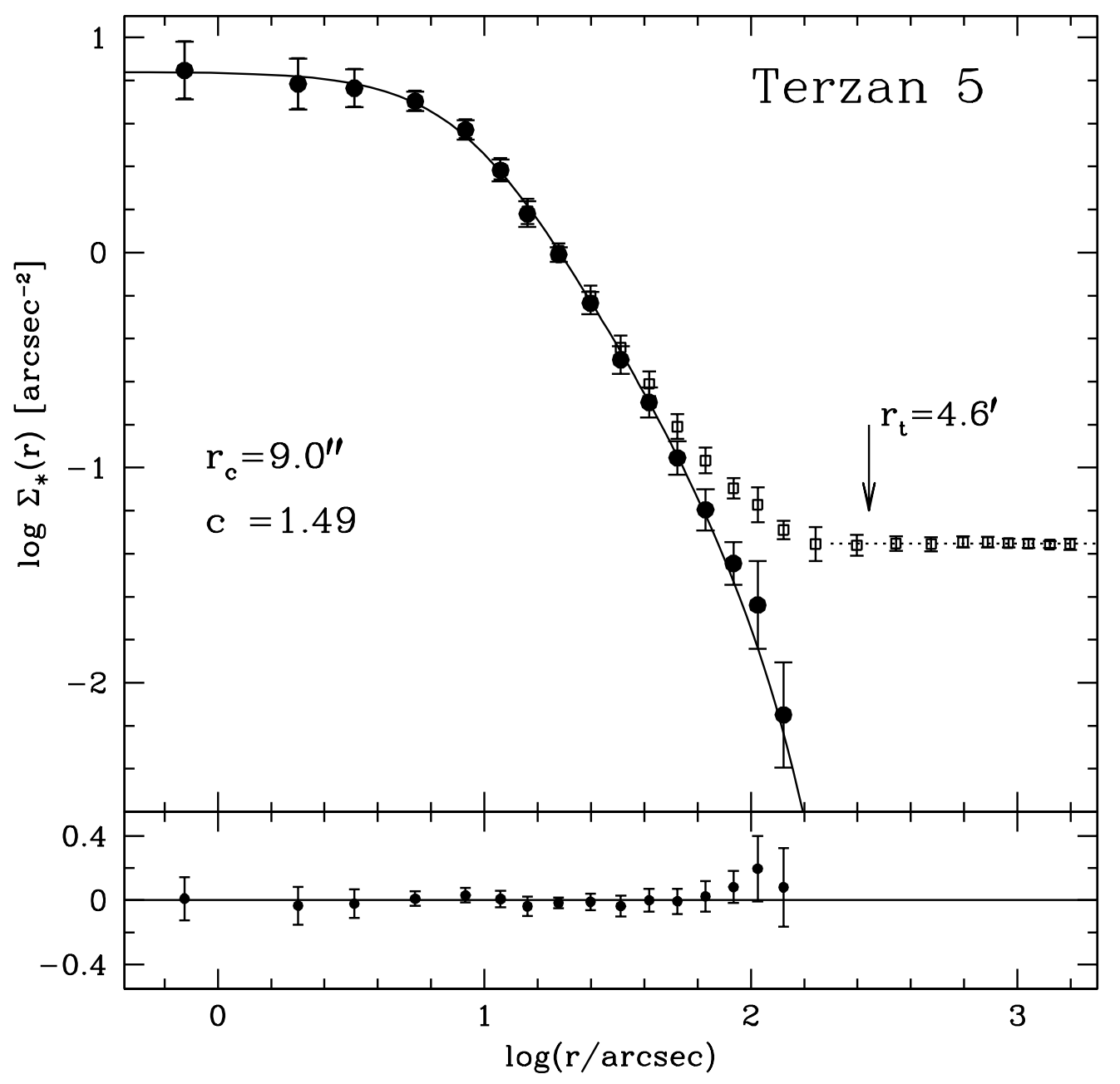

Fig. 3.- Newly determined star density profile of Terzan 5, obtained from resolved star counts in the combined photometric dataset. Empty squares represent the observed profile, while solid dots are obtained after subtraction of the field background density (marked with the dotted line). The best-fit single-mass King model is shown as a solid line and the corresponding structural parameters (core radius $r_{c}$, concentration $c$ and tidal radius $r_{t}$ ) are labelled in the figure. The lower panel shows the residuals between the observations and the fitted profile at each radial coordinate. 


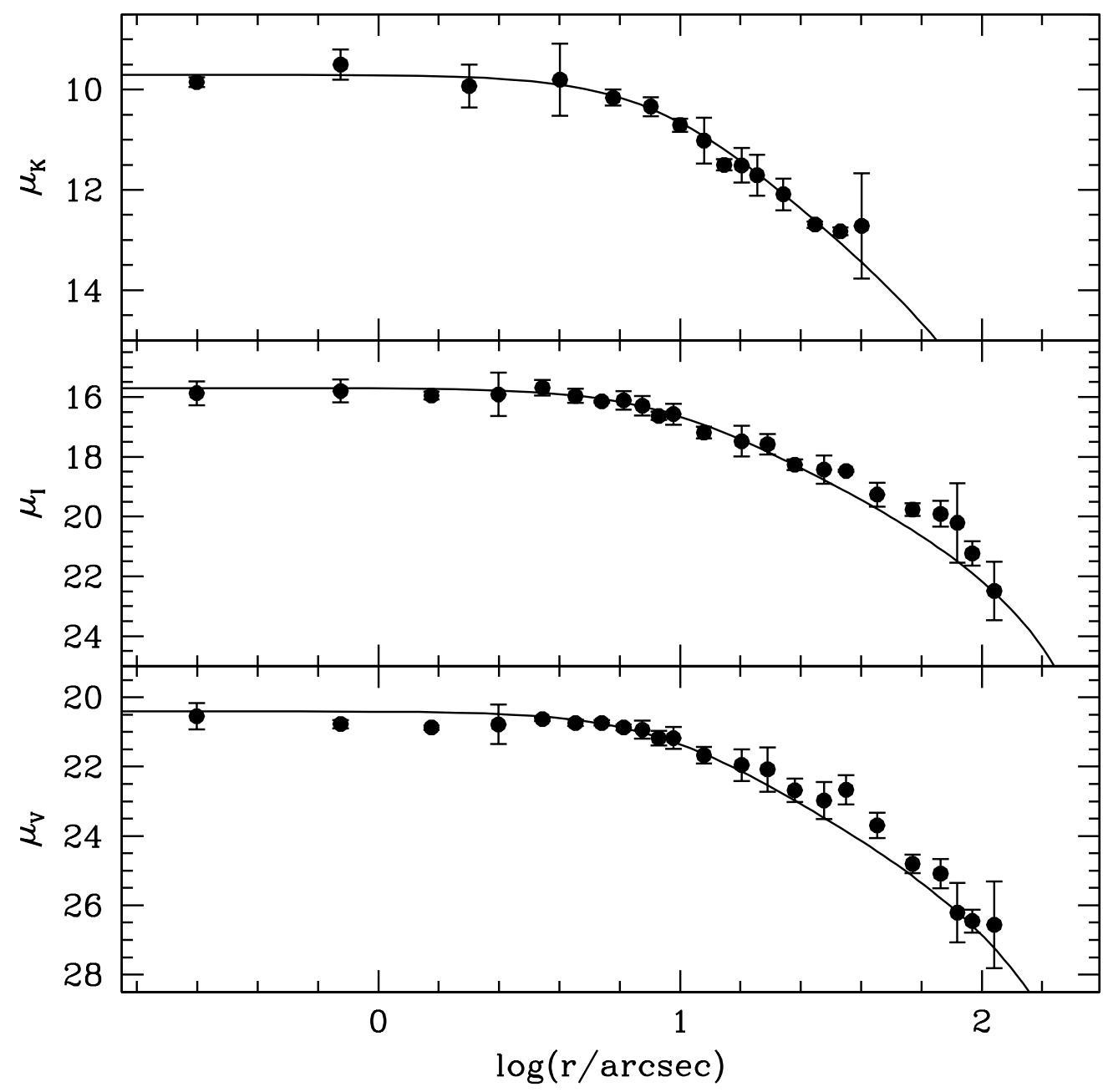

Fig. 4.- Surface brightness profile of Terzan 5 in three different filters, after background subtraction: $K$ band (upper panel), obtained from the MAD dataset, $I$ and $V$ bands (middle and lower panels), from the ACS sample. In each panel the solid line corresponds to the best-fit King model shown in Fig. 3 , 
Table 1. Structural parameters for Terzan 5

\begin{tabular}{ll}
\hline \hline Center of gravity & $\alpha_{\mathrm{J} 2000}=17^{\mathrm{h}} 48^{\mathrm{m}} 4.85^{\mathrm{s}}$ \\
& $\delta_{J 2000}=-24^{\circ} 46^{\prime} 44.6^{\prime \prime}$ \\
Reddening $^{\dagger}$ & $E(B-V)=2.38 \pm 0.055$ \\
Distance $^{\dagger}$ & $d=5.9 \pm 0.5 \mathrm{kpc}$ \\
Core radius & $r_{c}=9^{\prime \prime}=0.26 \mathrm{pc}$ \\
Concentration & $c=1.49$ \\
Total luminosity & $L_{\mathrm{bol}} \simeq 8 \times 10^{5} L_{\odot}$ \\
Total mass & $M_{\mathrm{T}} \simeq 2 \times 10^{6} M_{\odot}$ \\
Central mass density & $\rho_{0} \simeq 4.1 \times 10^{6} M_{\odot} / \mathrm{pc}^{3}$ \\
Central $K$-band SB & $\mu_{K}(0)=9.85 \mathrm{mag} / \operatorname{arcsec}^{2}$ \\
Central $I$-band SB & $\mu_{I}(0)=15.87 \mathrm{mag} / \operatorname{arcsec}^{2}$ \\
Central $V$-band SB & $\mu_{V}(0)=20.54 \mathrm{mag} / \operatorname{arcsec}^{2}$ \\
\hline \hline
\end{tabular}

Note. — ${ }^{\dagger}$ from Valenti et al. (2007) 